\title{
Editorial
}

\section{Complexity in Neural and Financial Systems: From Time-Series to Networks}

\author{
Tiziano Squartini (iD, ${ }^{1}$ Andrea Gabrielli, ${ }^{2}$ Diego Garlaschelli, ${ }^{1,3}$ Tommaso Gili (D), \\ Angelo Bifone, ${ }^{4}$ and Fabio Caccioli ${ }^{5}$
}

\author{
${ }^{1}$ IMT School for Advanced Studies Lucca, Lucca, Italy \\ ${ }^{2}$ Sapienza University of Rome, Rome, Italy \\ ${ }^{3}$ Instituut-Lorentz for Theoretical Physics, Leiden Institute of Physics, Leiden, Netherlands \\ ${ }^{4}$ Center for Neuroscience and Cognitive Systems, Istituto Italiano di Tecnologia, Rovereto, Italy \\ ${ }^{5}$ University College London, London, UK
}

Correspondence should be addressed to Tiziano Squartini; tiziano.squartini@imtlucca.it

Received 2 February 2018; Accepted 5 February 2018; Published 24 April 2018

\begin{abstract}
Copyright (C) 2018 Tiziano Squartini et al. This is an open access article distributed under the Creative Commons Attribution License, which permits unrestricted use, distribution, and reproduction in any medium, provided the original work is properly cited.
\end{abstract}

When can a system be unambiguously defined as "complex"? Although many real-world systems are believed to bear the signature of complexity, the question above remains unanswered. Our special issue aims at contributing to this ongoing discussion by collecting a number of studies tackling two aspects of complexity that have recently gained increasing attention: the temporal one and the structural one.

The seven papers composing this special issue offer an articulated overview of these topics by proposing novel techniques for the analysis of systems described by multiple timeseries (such as functional brain data, stock prices, and market indices) and networked interaction patterns. The choice of focusing on neural and financial systems is dictated by the importance that topics like the identification of precursors of stock market movements, the application of causality-testing techniques to brain data, and the definition of null models for the analysis of correlation matrices (only to mention a few) have gained in recent years.

In what follows, a brief overview of the contributions is provided.

H. Liao et al. contribute to the stream of research on "economic complexity," focusing on the International Trade Network and addressing the problem of forecasting the economic evolution of a country using predictors that go beyond standard economic quantities (such as GDP). In order to identify the best method, the authors compare three different metrics across a dataset ranging from 1962 to 2000. As a result, the "Fitness and Complexity" approach seems to outperform the competing techniques.

B. Podobnik et al. address another timely prediction problem, that is, the rise of EU right-wing populism in response to unbalanced immigration. In particular, the authors analyze the relationship between the percentage of right-wing (RW) populist voters in a given country, the prevalence of immigrants in the population of the same country, and the total immigration inflow into the entire EU over the last three years. They find that the increase in the percentage of RW voters substantially overcomes the percentage of immigration inflow. This result questions the role of EU institutions and calls for a deeper understanding of the EU citizens perception of the "globalization" topic.

A different, yet related, kind of prediction is the "linkprediction" problem, addressed by M.-Y. Zhou et al. The underlying hypothesis of any link prediction algorithm is that the likelihood of any two nodes to establish a connection depends on the number of "common" characteristics. The authors, however, propose a way of resorting link scores which enhances the precision of existing methods by penalizing to a lesser extent nodes characterized by lower similarity scores. 
Prediction problems are related to causality problems. Upon defining "causality" in an information-theoretic sense, T. Aste et al. investigate the performance of three methods (G-Lasso, Ridge, and LoGo) to detect causality links when short time-series are considered. A combination of filtering techniques and graphical modelling provides the best performance.

The same kind of filtering techniques is applied to investigate different types of dependency in financial multiplex networks. N. Musmeci et al. consider a four-layer multiplex defined by linear, nonlinear, tail, and partial correlations among a set of financial time-series. The structural evolution of this peculiar kind of network reveals changes associated with periods of financial stress, whose detection is enhanced by the multilayer character of the considered system.

Network techniques to analyze time-evolving, multiagent systems are also employed by A. Lombardi et al. to study the functional connectivity of the human brain. A novel framework is employed to quantify the synchronization of pairs of signals by exploiting the so-called cross-recurrence plots: community detection based on such a metric seems to outperform the usual one, based on the Pearson correlation coefficient.

Networks constitute also the support to explore "evolutionary" game theory. G. Cimini studies two different evolutionary dynamics in order to refine equilibria multiplicity of games of strategic substitutes and complements. When the latter are embedded in complex topologies, different behaviors are found for different classes of games. In particular, when coordination games on infinitely large scale-free networks are considered, equilibria arise for any value of the incentive to cooperate.

Tiziano Squartini Andrea Gabrielli Diego Garlaschelli Tommaso Gili Angelo Bifone Fabio Caccioli 


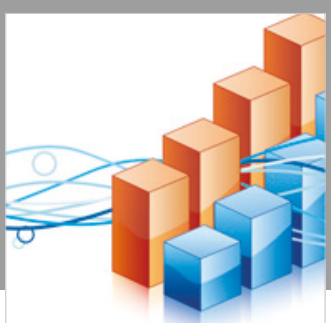

Advances in

Operations Research

\section{-n-m}
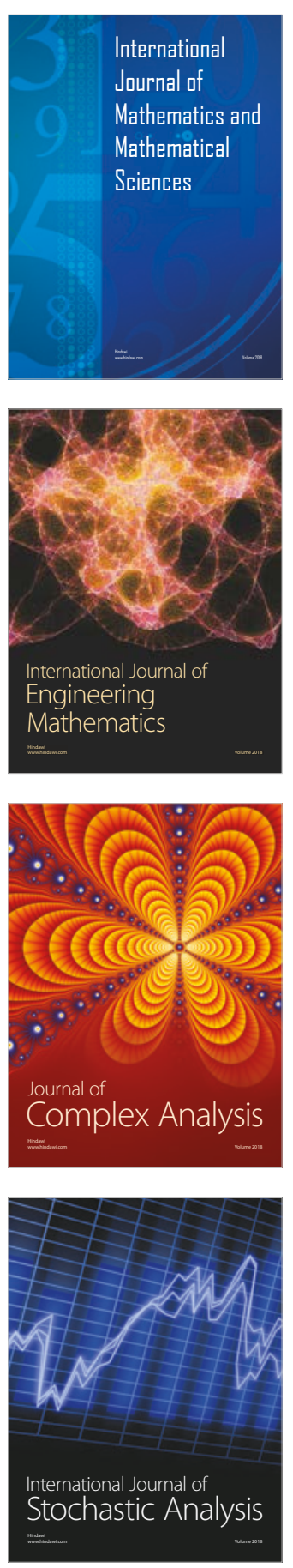
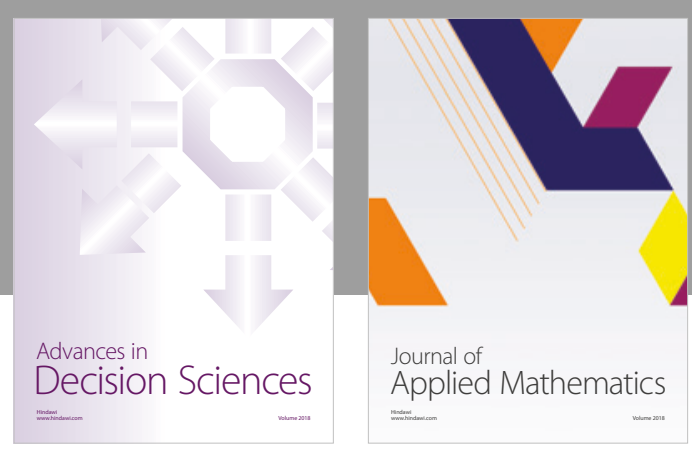

Journal of

Applied Mathematics
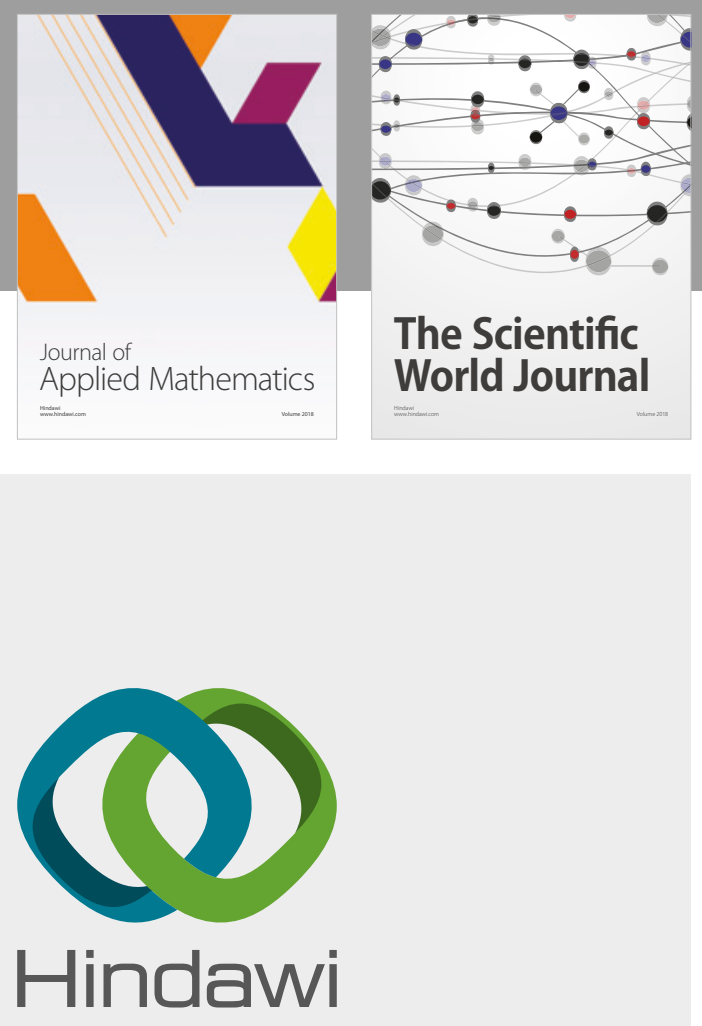

Submit your manuscripts at

www.hindawi.com

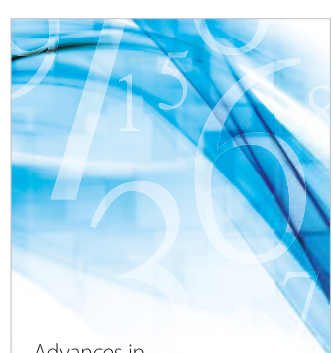

Advances in
Numerical Analysis
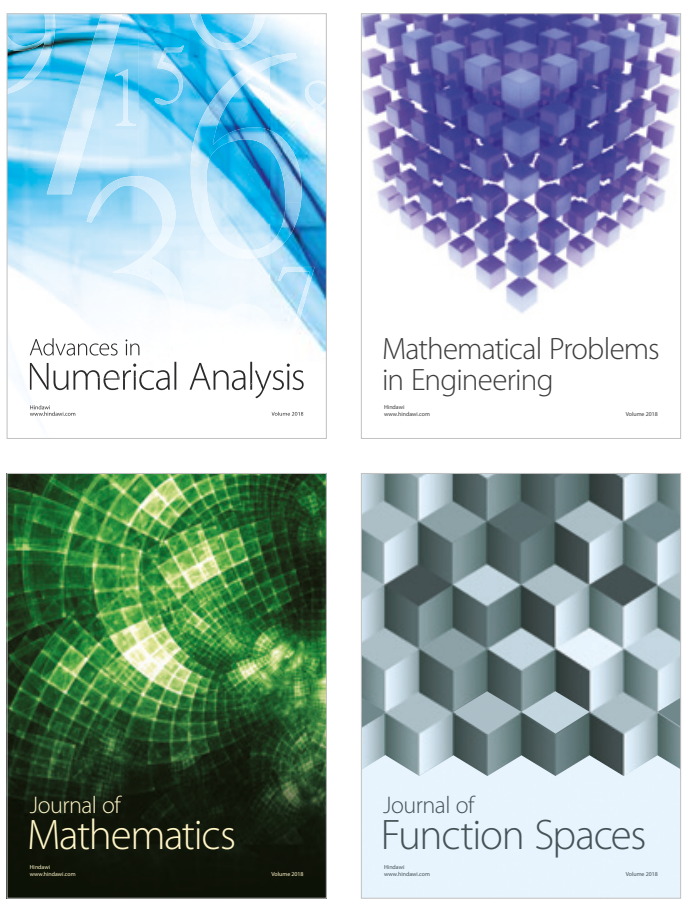

Mathematical Problems in Engineering

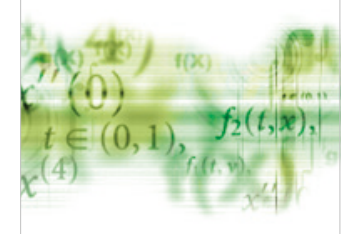

International Journal of

Differential Equations

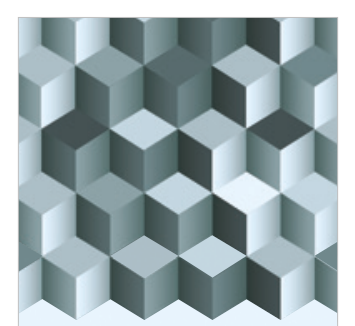

Journal of

Function Spaces
The Scientific

World Journal

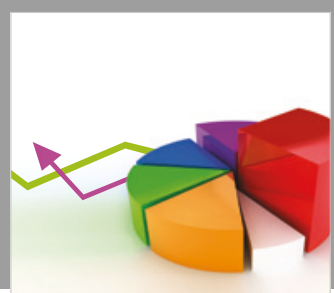

Journal of

Probability and Statistics
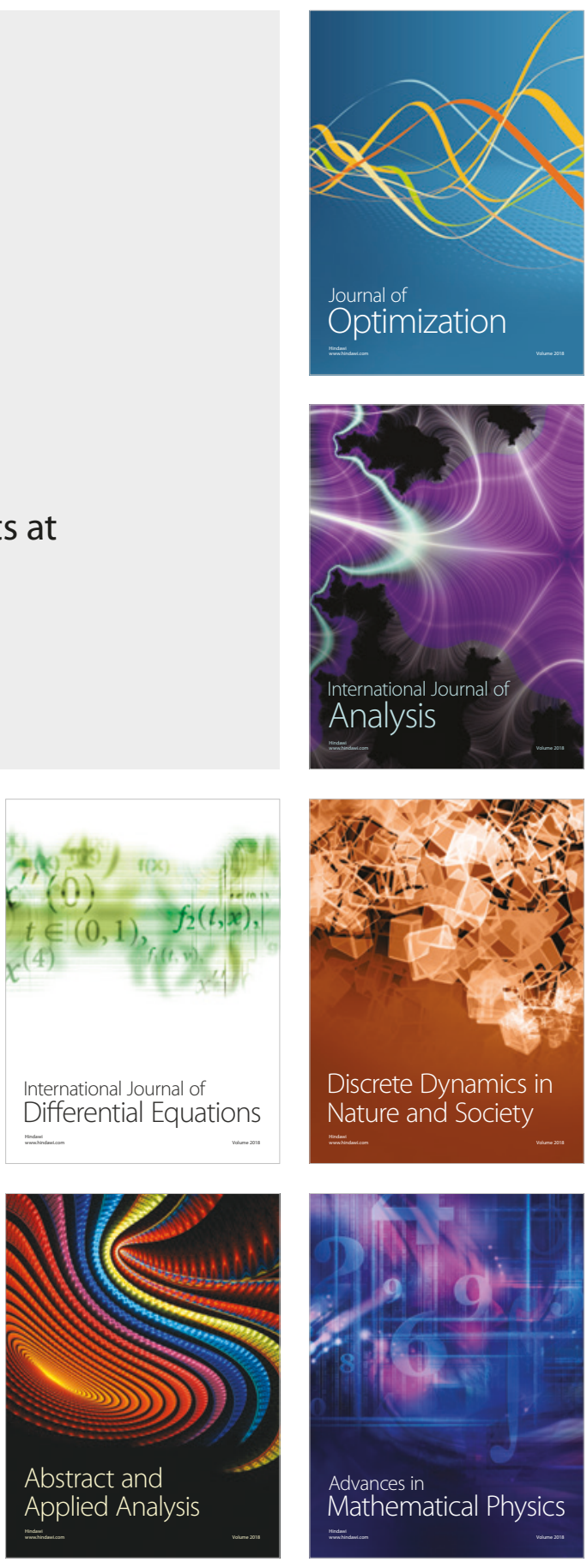